\title{
Highly nonlinear pulse splitting and recombination in a two-dimensional granular network
}

\author{
C. Daraio, ${ }^{1, *}$ D. Ngo, ${ }^{1}$ V. F. Nesterenko, ${ }^{2}$ and F. Fraternali ${ }^{3}$ \\ ${ }^{1}$ Aeronautics (GALCIT) and Applied Physics, California Institute of Technology, Pasadena, California 91125, USA \\ ${ }^{2}$ Mechanical and Aerospace Engineering Department, University of California at San Diego, La Jolla, California 92093-0411, USA \\ ${ }^{3}$ Department of Civil Engineering, University of Salerno, 84084 Fisciano (SA), Italy \\ (Received 11 March 2009; revised manuscript received 10 June 2010; published 8 September 2010;
} publisher error corrected 20 September 2010)

\begin{abstract}
The propagation of highly nonlinear signals in a branched two-dimensional granular system was investigated experimentally and numerically for a system composed of chains of spherical beads of different materials. The system studied consists of a double Y-shaped guide in which high- and low-modulus/mass chains of spheres are arranged in various geometries. We observed the transformation of a single or a train of solitary pulses crossing the interface between branches. We report fast splitting of the initial pulse, rapid chaotization of the signal and impulse redirection and bending. Pulse and energy trapping was also observed in the branches. Numerical analysis based on Hertzian interaction between the particles and the side walls of the guide was found in agreement with the experimental data, except for nonsymmetric arrangements of particles excited by a large mass striker.
\end{abstract}

DOI: 10.1103/PhysRevE.82.036603

\section{INTRODUCTION}

The study of the dynamical response of ordered low dimensional granular systems has recently received increasing attention, deriving from a broad range of interesting physical phenomena that have been unveiled [1-20]. The study of one-dimensional systems composed of a uniform chain of beads has allowed the theoretical and experimental discovery of a new highly nonlinear dynamic behavior in solids, supporting the formation and propagation of highly nonlinear compact solitary waves [1-3]. In addition the study of the highly nonlinear behavior at the interface between two highly nonlinear media allowed the discovery of interesting new dynamical effects arising from the local selforganization of the granules in close proximity to the interface $[4,5,10-12,17]$. Interfaces between two strongly nonlinear or linear/strongly nonlinear systems may exhibit "acoustic diode" behavior [11]. Strongly nonlinear systems composed of particles with different masses exhibit band gap effects that are tunable by initial precompression [19-21]. At higher dimensions, under static and quasistatic loading, structural features of the granular state include filamentary force (stress) chains, i.e., chains of granules with preferred contacts for force/pulse transmission [22-24]. In particular, dynamic stress chains can be a source of hot spot formation and were studied in $[25,26]$ for two-dimensional particle beds.

The experimental study of pulse propagation in these chains is essential for a better understanding of the response to dynamic impulse propagation in three-dimensional systems [27]. The passage of a highly nonlinear solitary wave from a one-dimensional chain of beads through a twodimensional geometrical bifurcation is also a matter of interest due to the non uniqueness of the outcome of this interaction based on conservation laws of energy and momentum. The outcome of this study may lead to possible engineering

\footnotetext{
*Corresponding author
}

PACS number(s): 05.45.Yv, 45.70.-n, 43.25.+y, 46.40.Cd

application in acoustic signals transmission and transformation. In this paper we describe the experimental observation of highly nonlinear solitary waves propagating through such interfaces and support our findings with a discrete numerical analysis of the systems. We study uniform assemblies composed of PTFE (polytetrafluoroethylene) and stainless steel beads, as well as heterogeneous structures composed of a combination of steel and polymer spheres including Parylene-C coated steel beads.

\section{EXPERIMENTAL SETUP AND RESULTS}

The experimental setup consists of a guide in which one chain contacts two others to allow splitting, redirecting and recombining the compressive pulse formed in the first chain by impact [27] (see Fig. 1 for the experimental and numerical setup). To create the two-dimensional system, we used a total number of 132 beads of uniform diameter $(4.76 \mathrm{~mm})$, composed of different materials: the high mass $(0.45 \mathrm{~g})$, high elastic modulus steel (nonmagnetic, 316 type) beads [11]; the small mass $(0.123 \mathrm{~g})$, low-modulus PTFE beads [14] and the high mass $(0.45 \mathrm{~g})$, low elastic modulus Parylene-C coated steel beads with slightly larger diameter $(4.86 \mathrm{~mm})$ [16]. The thickness of the Parylene-C coating was $50 \mu \mathrm{m}$. The properties of the materials composing the beads used in this study are summarized in Table I.

The beads were arranged in a horizontal PTFE guide with $\sim 5 \mathrm{~mm}$ diameter half-cylindrical grooves carved in a diamondlike double-Y shape, with bifurcation angles of $60^{\circ}$ between the two branches [see Fig. 1(a) for a schematic]. Four calibrated piezosensors were embedded inside particles in the system as described in [3-5,14-17] [see Fig. 1(b)] and connected to a Tektronix oscilloscope, allowing for calculating the signal's speed and monitoring the wave's shape. Pulses were generated with swinging spherical steel strikers of different masses ( 0.45 and $5.33 \mathrm{~g})$ at various impact velocities $(0.1-1.0 \mathrm{~m} / \mathrm{s})$.

The results of a first set of experiments performed on uniform systems composed of all stainless steel beads are 


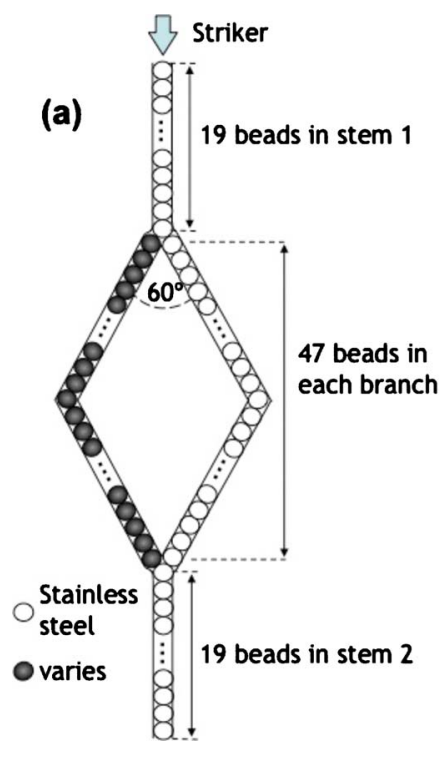

(b)

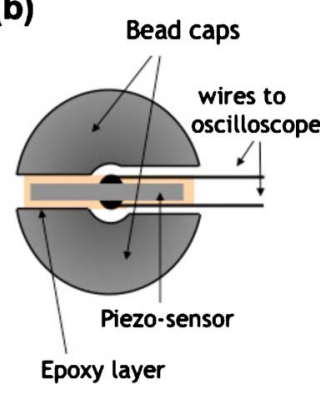

FIG. 1. (Color online) (a) Experimental setup showing the diamond shaped guide and indicating the number of beads composing each portion of the system. (b) Schematic of the composition of a particle with an embedded piezogauge.

presented in Fig. 2(a) and 3(c) and for all PTFE beads in Fig. 3(a). Sensors were positioned in each one of the branches as pointed by the lines in the corresponding curves (e.g., 12th in stem 1 means the sensor was placed in the 12th particle counting from the impacted end, and fourth in branch means a sensor placed in the fourth particle counting from the bifurcated interface), to monitor the shape and duration of the pulses before and after the bifurcated interface. Waves were excited by a steel striker with a mass $0.45 \mathrm{~g}$ or $5.33 \mathrm{~g}$ as indicated in the captions of the corresponding figures.

A second set of experiments was performed on composite systems with asymmetric branches where the stainless steel beads in one of the branches were replaced with polymer based (low modulus and/or low mass) particles [refer to Fig. 1(a) where the dark beads represent, in this case, the PTFE particles and the white beads the stainless steel one]. This set up was chosen to analyze the wave behavior in highly nonlinear systems having a completely new type of interfaces, connecting bifurcating branches with different masses and signal's speeds. This arrangement is important to understand the fundamental mechanisms governing the responses of force chains in three-dimensional systems and also to create practical devices for signal phase shifting, pulse scrambling and energy trapping within one of the branches. Results obtained in the stainless steel/PTFE based system are shown in Fig. 4(a), where a $0.45 \mathrm{~g}$ striker was used to excite a single
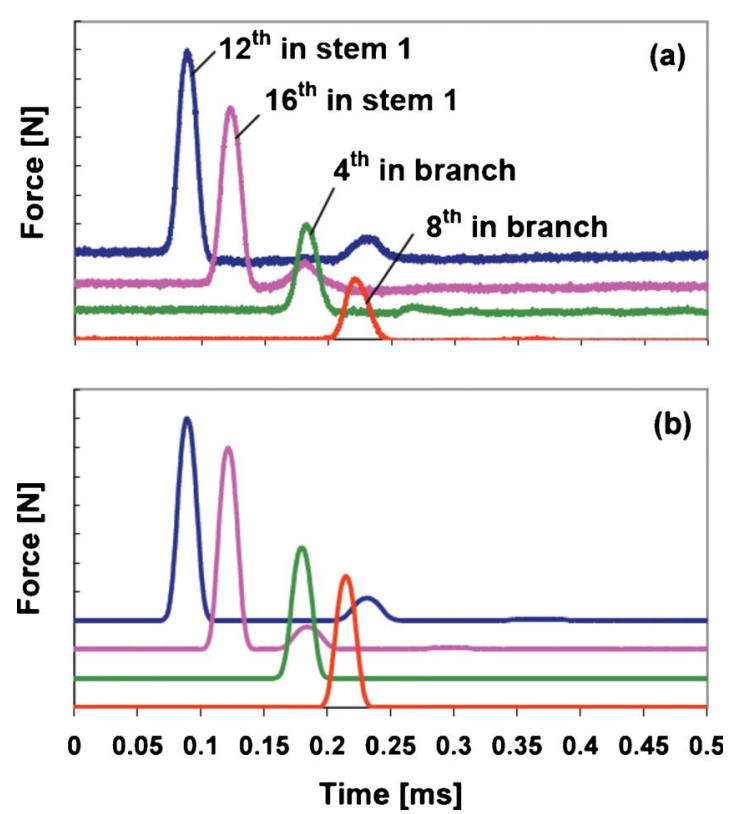

FIG. 2. (Color online) Highly nonlinear waves propagating in the Y-shape guide composed of all stainless steel particles. (a) Experimental data corresponding to the transformation of a solitary wave excited by an identical steel striker traveling at $\sim 0.585 \mathrm{~m} / \mathrm{s}$ in the first horizontal branch. The $y$-axes scale is $2.5 \mathrm{~N}$ per division. (b) Corresponding numerical results. The $y$-axes scale is $2.5 \mathrm{~N}$ per division.

solitary wave in the first 19 beads before the interface.

The last system tested was arranged similarly to the previous case, with Parylene-C coated steel beads replacing the PTFE particles in one of the system's branches. In this case the mass of the particles was maintained uniform, and only the value of the elastic modulus was varied between the two branches. The experimental results for a single solitary wave and a train of solitary waves initially excited in the system using strikers with different masses are shown in Figs. 5(a) and 6(a) correspondingly.

\section{NUMERICAL MODELING}

The dynamics of the experimental system under considerations is necessarily affected by irreversible phenomena, like e.g., friction and plasticity. Nevertheless we hereafter adopt a simplified nondissipative model, in agreement with the standard models in the literature (cf. [3]). It was shown that the use of an approach without dissipation is justified for the explanation of the main features of strongly nonlinear waves in systems composed by a relatively small number of grains

TABLE I. Properties of the materials composing the highly nonlinear systems tested.

\begin{tabular}{lcccc}
\hline \hline & $\begin{array}{c}\text { Elastic modulus } \\
(\mathrm{GPa})\end{array}$ & Poisson ratio & $\begin{array}{c}\text { Density } \\
\left(\mathrm{kg} / \mathrm{m}^{3}\right)\end{array}$ & $\begin{array}{c}\text { Bead mass } \\
(\mathrm{g})\end{array}$ \\
\hline Stainless steel beads [14,16] & 193 & 0.30 & 8000 & 0.45 \\
PTFE beads [14] & 1.46 & 0.46 & 2200 & 0.123 \\
Parylene-C coating [16] & 15 & 0.38 & 1289 & 0.45 \\
\hline \hline
\end{tabular}



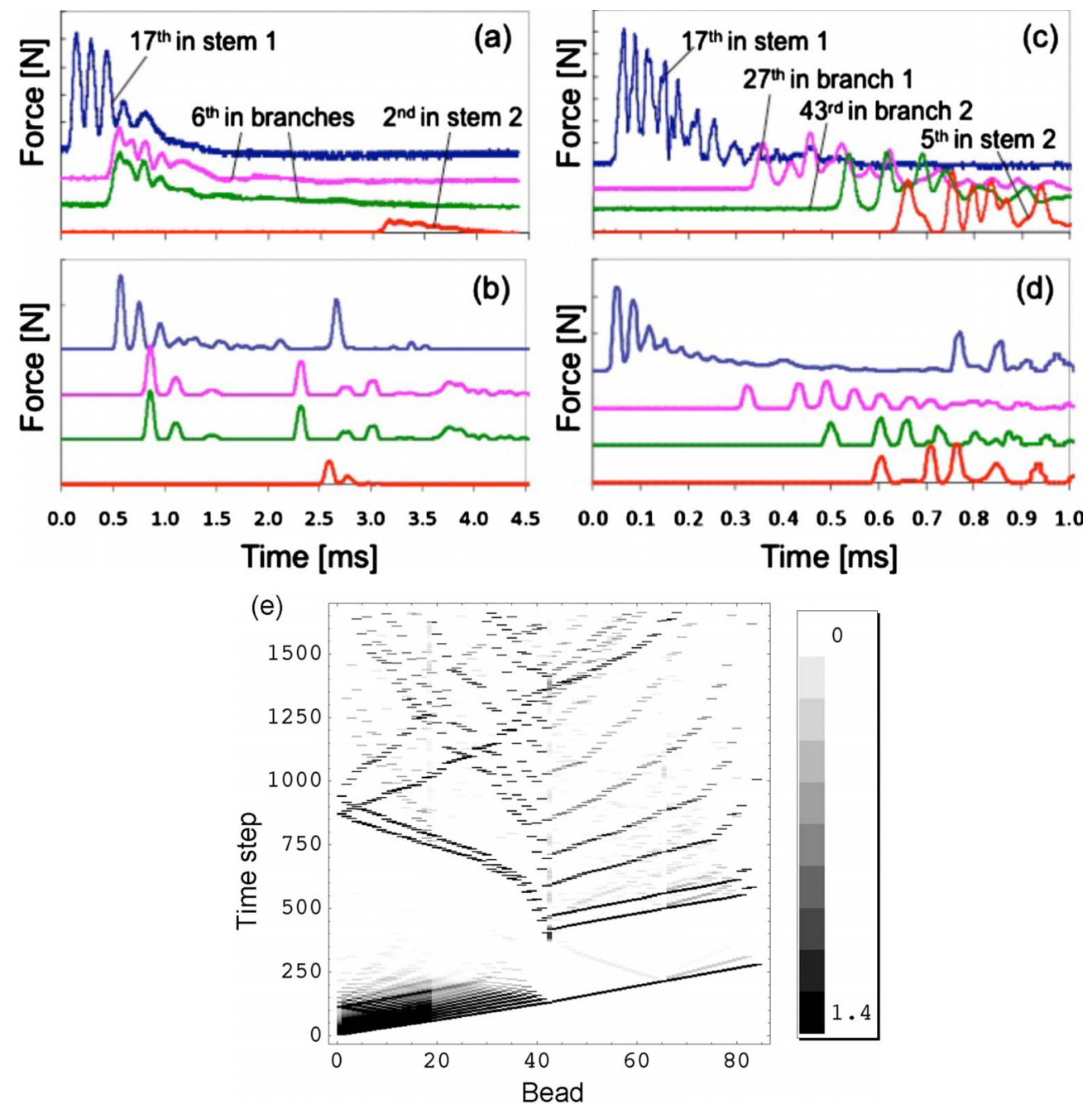

FIG. 3. (Color online) Trains of highly nonlinear waves propagating in uniform systems composed of all PTFE and all stainless steel beads showing the experimental $(\mathrm{a}, \mathrm{c})$ and corresponding numerical data $(\mathrm{b}, \mathrm{d})$. In the latter, the curves plotted correspond to the same sensor position as in experiments. (a) Waves excited by a $0.45 \mathrm{~g}$ striker in a PTFE based system at $\sim 0.25 \mathrm{~m} / \mathrm{s}$ impact velocity. The $y$-axes scale for all curves is $0.2 \mathrm{~N}$ per division. (b) Numerical simulations corresponding to (a). The $y$-axes scale for all curves is $0.5 \mathrm{~N}$ per division. (c) Waves excited by a $5.33 \mathrm{~g}$ steel striker in a stainless steel based system at $\sim 0.18 \mathrm{~m} / \mathrm{s}$. The $y$-axes scale for the top curve is $2 \mathrm{~N}$ per division and $1 \mathrm{~N}$ per division for the other curves. (d) Numerical simulations corresponding to (c). The $y$-axes scale for all curves is $5 \mathrm{~N}$ per division. The curve with the fastest impulse propagation corresponds to the signal recorder from the branch composed of steel beads. The top curves are shifted on different divisions to ease readability. (e) Density plots of numerically computed particle forces (N) in the subsystem of Figs. 3(c) and 3(d) composed of stem 1-steel branch (either branch 1 or branch 2, due to symmetry) and stem 2 (full steel system). The horizontal axis is labeled according to particle site along each branch and the vertical axis gives the time step number (each time step $=3 \times 10^{-6} \mathrm{~s}$ ).

$[4-6,9,11,12,14-20,27-29]$. In detail, we model each setup described in the previous section as a system of $N$ spheres $\mathcal{P}_{1}, \ldots, \mathcal{P}_{N}$ interacting through Hertzian unilateral potentials [3]. A schematic diagram showing the notation used for the modeling is depicted in Fig. 7.

Let us denote the particle centers of mass by $G_{1}, \ldots, G_{N}$; the correspondent Cartesian coordinates by $\left(x_{1}, y_{1}\right), \ldots,\left(x_{N}, y_{N}\right)$; the particle masses by $m_{1}, \ldots, m_{N}$; and the particle radii by $r_{1}, \ldots, r_{N}$. We collect the Cartesian coordinates $\left(x_{1}, y_{1}\right), \ldots,\left(x_{N}, y_{N}\right)$ into a numerical vector $\mathbf{q}$ of generalized coordinates, which owes $M=2 N$ components $q_{1}, \ldots, q_{M}$, such that $q_{2 i-1}=x_{i}$, and $q_{2 i}=y_{i}$, for $i=1, \ldots, N$. We also define a numerical vector $\mathbf{p}$ of $M$ generalized momenta $p_{1}, \ldots, p_{M}$, which are defined as follows: $p_{2 i-1}=m \dot{x}_{i} ; p_{2 i}$ $=m \dot{y}_{i},(i=1, \ldots, N)$, where the dots over variables denote time derivatives.

Given an arbitrary couple of particles $\left(\mathcal{P}_{i}, \mathcal{P}_{j}\right)$, it is convenient to introduce the two points $P_{i j}$ and $P_{j i}$, which coincide with the intersection of the segment $G_{j}-G_{i}$ with the boundary of $\mathcal{P}_{i}$, and the intersection of $G_{i}-G_{j}$ with the boundary of $\mathcal{P}_{j}$, respectively (Fig. 7). We let $\underline{x}_{i}, \underline{x}_{j}, \underline{y}_{i}$, and $\underline{y}_{j i}$ indicate the positions vector of $G_{i}, G_{j}, P_{i j}$; and $P_{j i}$, respectively (Fig. 7). The following scalar quantities

$$
\delta_{i j}=-\left(\underline{y}_{j i}-\underline{y}_{i j}\right) \cdot \underline{n}_{i j}
$$

indicate the particle relative displacements $(i, j$ $=1, \ldots, N ; i \neq j)$, which are negative when $\mathcal{P}_{i}$ and $\mathcal{P}_{j}$ form a 

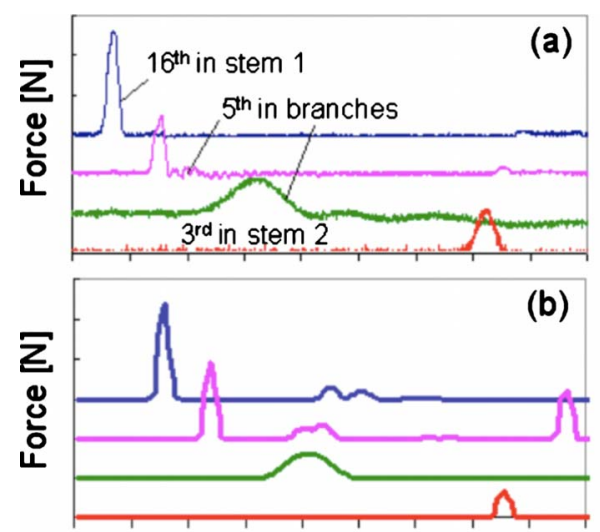

$\begin{array}{lllllllllll}0.0 & 0.1 & 0.2 & 0.3 & 0.4 & 0.5 & 0.6 & 0.7 & 0.8 & 0.9\end{array}$ Time [ms]

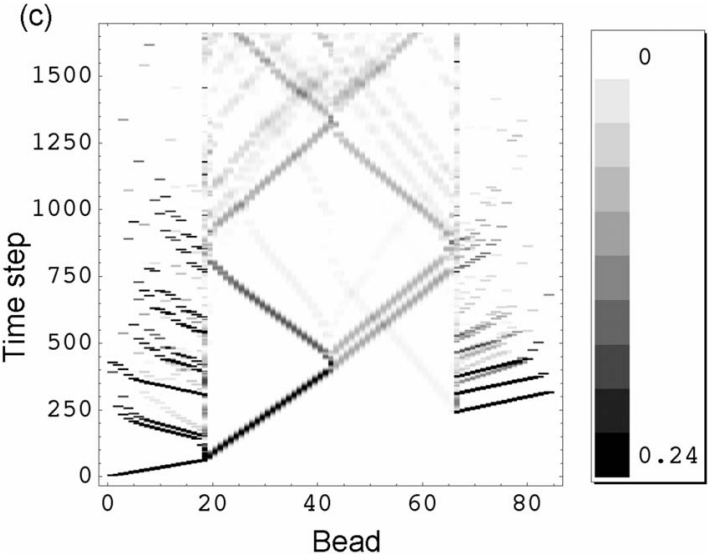

FIG. 4. (Color online) Highly nonlinear waves propagating in the diamond-shaped holder having one of the branches filled with PTFE beads and the rest of the structure composed of stainless steel particles. (a) Experimental data corresponding to the transformation of a solitary wave excited by a $0.45 \mathrm{~g}$ striker traveling at $\sim 0.20 \mathrm{~m} / \mathrm{s}$ in the first horizontal branch. The $y$-axes scale for the top two curves is $2 \mathrm{~N}$ per division, while it is $0.2 \mathrm{~N}$ per division for the two lower curves. (b) Corresponding numerical results. The $y$-axes scale is $2 \mathrm{~N}$ per division except for the third curve from the top (green) where it is $0.4 \mathrm{~N}$ per division. The curve with the fastest impulse propagation corresponds to the signal recorder from the branch composed of steel beads. The top curves are shifted on different divisions to ease readability. (c) Density plots of numerically computed particle forces (N) in the subsystem of Figs. 4(a) and 4(b) composed of stem 1-PTFE branch and stem 2. The horizontal axis is labeled according to particle site along each branch and the vertical axis gives the time step number (each time step $=3 \times 10^{-6} \mathrm{~s}$ ).

gap and positive when instead such particles penetrate each other. Here, $\underline{n}_{i j}$ is the unit vector of the direction $G_{j}-G_{i}$ and $(\cdot)$ denotes the scalar product between vectors.

Using the settings above, the dynamics of the system in Fig. 1 is described by the Hamiltonian [30]

$$
H=\sum_{i=1}^{N}\left[\frac{\left(p_{2 i-1}\right)^{2}+\left(p_{2 i}\right)^{2}}{2 m_{i}}\right]+\sum_{i=1}^{N} \sum_{j>i} V_{i j}\left(\delta_{i j}\right),
$$

where $V_{i j}$ denotes the interaction potential associated with the couple of particles $\left(\mathcal{P}_{i}, \mathcal{P}_{j}\right)$. Following [1-3], we describe
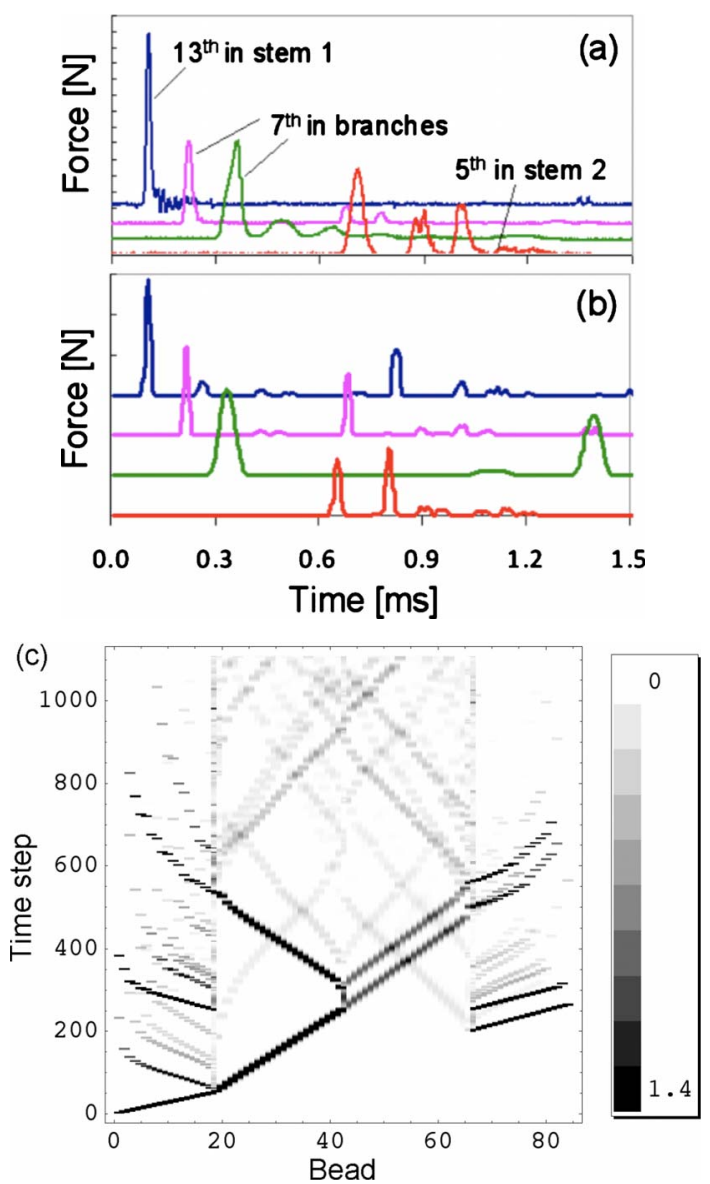

FIG. 5. (Color online) Highly nonlinear waves propagating in the diamond-shaped holder having one of the branches filled with Parylene-C coated steel beads and the rest of the structure composed of bare stainless steel particles. System excited by a $0.45 \mathrm{~g}$ striker traveling at $\sim 0.50 \mathrm{~m} / \mathrm{s}$. (a) Experimental data, the $y$-axes scale is $2 \mathrm{~N}$ for the two top most curves, $0.4 \mathrm{~N}$ per division for the third curve and $0.1 \mathrm{~N}$ for the bottom curve. (b) Numerical simulations, the $y$-axis scale is $5 \mathrm{~N}$ per division for the two topmost curves, $1.25 \mathrm{~N}$ for the third (green) and $2.5 \mathrm{~N}$ for the bottom (red) curve. The curve with the fastest impulse propagation corresponds to the signal recorder from the branch composed of steel beads. The top curves are shifted on different divisions to ease readability. (c) Density plots of numerically computed particle forces $(\mathrm{N})$ in the subsystem of Figs. 5(a) and 5(b) composed of stem 1-Parylene-C coated branch and stem 2 . The horizontal axis is labeled according to particle site along each branch and the vertical axis gives the time step number (each time $\mathrm{step}=3 \times 10^{-6} \mathrm{~s}$ ).

particle interactions through the unilateral Hertz contact law

$$
V_{i j}=\frac{2}{5} \alpha_{i j}\left(\delta_{i j}^{+}\right)^{5 / 2}
$$

where $\delta_{i j}^{+}$is the positive part of $\delta_{i j}$ and

$$
\alpha_{i j}=\frac{4 E_{i} E_{j} \sqrt{\frac{r_{i} r_{j}}{r_{i}+r_{j}}}}{3 E_{i}\left(1-v_{j}^{2}\right)+3 E_{j}\left(1-v_{i}^{2}\right)},
$$

$E_{i}$ and $v_{i}$ being the Young modulus and the Poisson ratio of $\mathcal{P}_{i}$, respectively (Table I). 

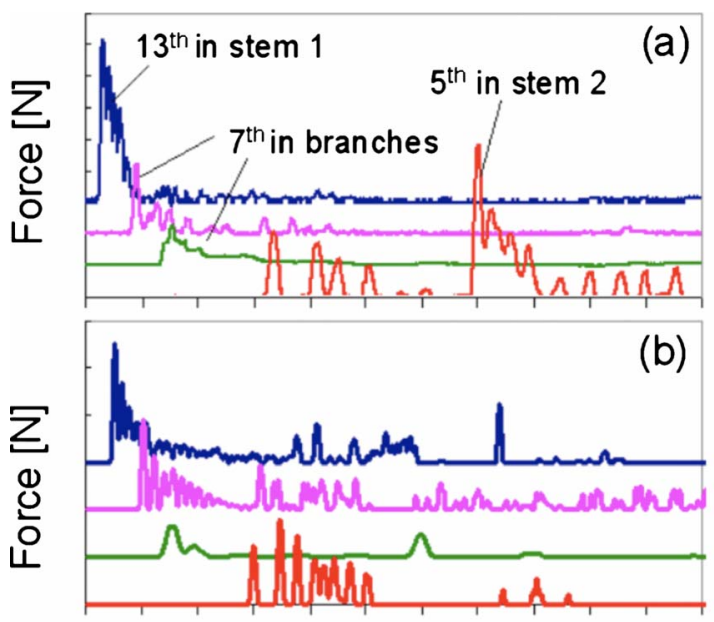

$\begin{array}{lllllllllllll}0.0 & 0.2 & 0.4 & 0.6 & 0.8 & 1.0 & 1.2 & 1.4 & 1.6 & 1.8 & 2.0 & 2.2\end{array}$ Time $[\mathrm{ms}]$

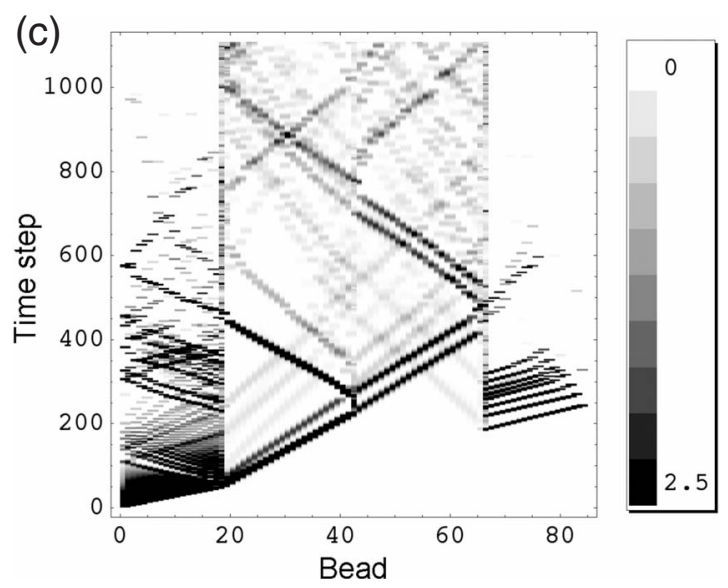

FIG. 6. (Color online) Highly nonlinear waves propagating in the diamond-shaped holder having one of the branches filled with Parylene-C coated steel beads and the rest of the structure composed of bare stainless steel particles. System excited by a $5.33 \mathrm{~g}$ striker traveling at $\sim 0.35 \mathrm{~m} / \mathrm{s}$. (a) Experimental data, the $y$-axes scale for the three topmost curves is $5 \mathrm{~N}$ per division and $0.5 \mathrm{~N}$ per division for the last (bottom) curve. (b) Numerical simulations, the $y$-axis scale is $10 \mathrm{~N}$ for all curves except the bottom (red) curve that is $5 \mathrm{~N}$. The curve with the fastest impulse propagation corresponds to the signal recorder from the branch composed of steel beads. The top curves are shifted on different divisions to ease readability. (c) Density plots of numerically computed particle forces $(\mathrm{N})$ in the subsystem of Figs. 6(a) and 6(b) composed of stem 1-Parylene-C coated branch and stem 2 . The horizontal axis is labeled according to particle site along each branch and the vertical axis gives the time step number (each time step $=3 \times 10^{-6} \mathrm{~s}$ ).

For the numerical treatment of the Parylene-C coated particles we considered that the particle's stainless steel core behaved as a rigid body and that the depth of the deformed contact zone was always smaller than the thickness of the coated layer. At time zero the particles are assumed to be in point contact with each other, reproducing the experimental setup for the various geometries. We model the Y-shaped guides as particles with infinitely large radii ("walls") and materials properties of PTFE. In other words, the $i$ th guide is
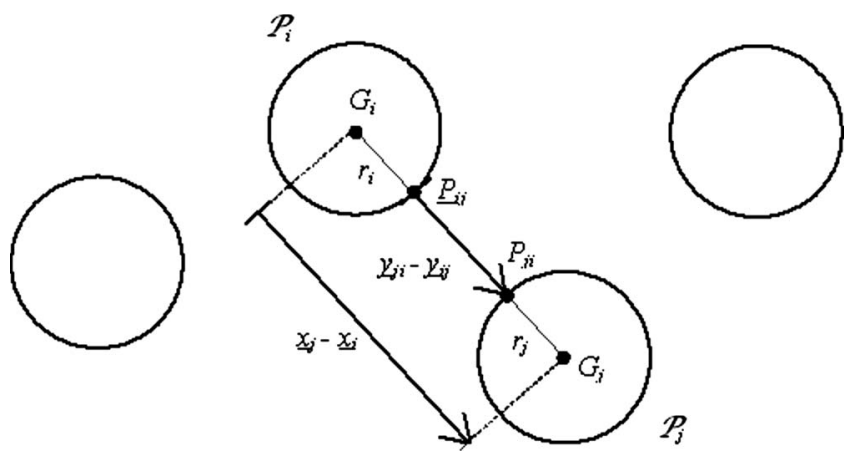

FIG. 7. Schematic showing the notation used in the numerical modeling.

regarded as a particle with constant generalized coordinates $\left(x_{i}, y_{i}\right)$ during the motion; $\underline{n}_{i j}$ always coincident with the outward normal to the guide (for any $j$ ); having $E_{i}$ and $v_{i}$ coincident with those of PTFE beads in Table I; and $r_{i} \rightarrow \infty$. For each particle and at each instant, we compute the force

$$
\bar{F}_{i}=\frac{1}{2} \sum_{j} \frac{\partial V_{i j}}{\partial \delta_{i j}}\left|\underline{n}_{i j} \cdot \underline{k}_{i}\right|,
$$

where $k_{i}$, is the unit vector of current displacement vector of $\mathcal{P}_{i}$. Such a quantity can be usefully compared with force measurements performed through embedded piezosensors. The Hamilton equations of motion of the system [30]

$$
\dot{p}_{m}=-\frac{\partial H}{\partial q_{m}}, \quad \dot{q}_{m}=\frac{\partial H}{\partial p_{m}}
$$

form a system of $2 M$ first order differential equations in the unknowns $q_{m}, p_{m}$, to be solved with the aid of the initial conditions $p_{m}(t=0)=p_{m}^{(0)}, q_{m}(t=0)=q_{m}^{(0)}$, for given $p_{m}^{(0)}$ and $q_{m}^{(0)}(m=1, \ldots, M)$. Equations (6) were solved numerically through a Runge-Kutta integration scheme [31], assuming a time integration step $\Delta t=10^{-6} \mathrm{~s}$, which ensured relative errors lower than $10^{-5}$ in the total energy conservation $(E=H$ $=T+V=$ const $)$. The results of numerical calculations are presented in Figs. 2(b), 3(b), 3(d), 3(e), 4(b), 4(c), 5(b), 5(c), 6(b), and 6(c) coupled with corresponding experimental results for convenient comparison.

\section{DISCUSSION}

We begin discussing the behavior of a single highly nonlinear solitary wave crossing a $Y$-shaped interface in a uniform system to understand qualitatively and quantitatively how a single solitary wave splits into two symmetric branches with similar particles. In this particular system, the behavior of the solitary wave may be predicted using conservation laws of momentum and energy before and after the interface. This can be achieved by using the concept of "effective mass" of a solitary wave equal to about 1.4 times the mass of one particle in the chain $[9,28]$, and by assuming that only single solitary waves represent the reflected and transmitted signals in each branch.

From the conservation laws point of view this case is analogous to the well-known behavior of a billiard ball hit- 
ting with velocity $v_{\mathrm{p}}$ two other similar balls of equal mass being at rest and arranged in a geometry similar to Y-junction. At the same time there is a qualitative difference between the impact of three balls and the interaction of a highly nonlinear solitary wave with a $Y$-shaped symmetric junction between uniform chains. It is due to the fact that energy and momentum conservation laws result in uniquely defined velocities of the three billiard balls after their interaction, while there are infinite numbers of combinations of transmitted and reflected solitary waves which can satisfy conservation laws in the latter case. For example, if we assume that incident solitary wave with linear momentum $I_{0}$ resulted in a single reflected solitary wave with linear momentum $I_{1}$ and $n$ solitary waves in each of the branches with equal amplitudes and linear momentum $I_{2}$ then, for this specific symmetric arrangement of balls [Fig. 1(a)], these values are equal to

$$
I_{1}=\frac{3 n-2}{2+3 n} I_{0}, \quad \text { and } \quad I_{2}=\frac{2 \sqrt{3}}{2+3 n} I_{0}
$$

To address this issue we compare experimental data with numerical simulations of the discrete elements and with predictions followed from conservation laws [Eqs. (7), $n=1$ ]. In this case, we used a separate experimental setup consisting of only the first half of the diamond-shaped guide. We filled three branches (the incoming stem-stem 1, and two branches after the Y-interface) with 65 identical stainless steel particles (19 particles in the stem 1 and 23 particles in each branch). Four particles with embedded sensors were placed at positions 12th and 16th in stem 1 and at positions 4th and 8th in one of the branches to measure the signals' speed and amplitude. A single solitary wave was created in stem 1 by impacting the first particle with a striker identical to the particles in the system. Figures 2(a) and 2(b) show the experimental data and the corresponding numerical results in the case when the chain was excited by the striker with a $0.585 \mathrm{~m} / \mathrm{s}$ impact velocity (the impact velocity was measured using an optical velocimeter connected to a digital oscilloscope).

It is important to note that after the interaction of the incident solitary with the Y-junction interface, two identical single solitary waves were transmitted in the branches. In addition, a reflected solitary wave was observed propagating backward from the junction in stem 1. This reflected wave was evident both in experiments and numerical calculations (see Fig. 2, in which the top two lines, represent the sensors placed in stem 1). No other significant signals were observed in experiments and calculations. The incident solitary wave speed $V_{i}$ in stem 1 , the transmitted solitary wave speed $V_{t}$ in the branches and the reflected solitary wave speed $V_{r}$ in stem 1 were calculated from the experimental data: $V_{i}$ $=566.96 \mathrm{~m} / \mathrm{s}, V_{t}=506.64 \mathrm{~m} / \mathrm{s}$ and $V_{r}=415.92 \mathrm{~m} / \mathrm{s}$ result ing in the ratios $V_{t} / V_{i}=0.894$ and $V_{r} / V_{i}=0.734$. The corresponding velocity ratios obtained from the numerical simulations were $V_{t} / V_{i}=0.917$ and $V_{r} / V_{i}=0.688$ in good agreement with the experimental results. It is remarkable that the solitary wave transmission across the interface was accomplished on the space scale of only a few particles (4) from the junction.

The maximum force $\left(F_{\mathrm{m}}\right)$ in a solitary wave is related to the linear momentum $(I)$ of the solitary wave as $F_{\mathrm{m}} \sim I^{6 / 5}[3]$. Based on linear momentum conservation laws [Eq. (7)] the ratio of the maximum force in the incident solitary wave over the maximum force in the transmitted solitary wave (assuming $n=1$ in each one of the branches) should be equal to 1.6. Accordingly, the ratio of the maximum force in the incident wave over the maximum force in the reflected wave should be 6.9 . These ratios are in excellent agreement with the ratios obtained comparing the incident, transmitted and reflected solitary waves in numerical calculations [Fig. 2(b)]. In experiments, the ratio of the maximum force in the incident solitary wave (measured in the 16th particle from the striker side) over the maximum force in the transmitted solitary wave (measured in the 4th particle after the interface) was found to be equal to 2. Similarly, the ratio of the maximum force in the incident wave over the maximum force in the reflected wave (measured in the 16th particle from the striker side) was found to be 8.4. Such higher values found in experiments could be explained by the participation of the guide walls in the elastic interaction of the incident wave with the Y-junction, by dissipation and/or by asymmetries in the contacts geometry at the junction. This agreement between the numerical data and experimental results with the predictions based on conservation laws [Eqs. (7)] suggests that it is possible to consider solitary waves as quasiparticles. Similar conclusions can be drawn based on the measured solitary wave speeds, considering the proportionality between solitary wave speed and momentum as $V_{\mathrm{s}} \sim I^{1 / 5}$ [3]. These relations can be employed to tune the behavior of the solitary wave splitting at the Y-junction for specific applications (like signal redirection, energy trapping and for the design of protective systems).

The behavior of a train of solitary waves interacting with symmetric, identical branches and later merging back into a single chain is presented in Figs. 3(a) and 3(b) for PTFE and in Fig. 3(c) and 3(d) for stainless steel beads. For the PTFE based system [refer to Fig. 3(a) for experiments and Fig. 3(b) for the corresponding numerical analysis], the wave train, after crossing the first interface, appears to split in two identical trains, with amplitude about half of its initial one. It is interesting to notice that the presence of dissipation in experiments tends to attenuate the propagation of the reflected pulses from the bends and junctions, though visible in the numerical results [Fig. 3(b)]. The ability of the waves in the branches to recombine together in the final stem demonstrates the capability of the pulses in such system to undergo $150^{\circ}$ and $120^{\circ}$ bends despite energy losses and interaction with the walls. Similar results were observed also for the stainless steel based system [Figs. 3(c) and 3(d)], where the presence of reflections from the walls, in particular deriving from the pulse particles interaction at the bends, is more evident (refer to the irregular pulses in Figs. 3(c) and 3(d) for the 27th and 43rd particles in the branches). A numerical density plot of the forces traveling in the full steel system is reported in Fig. 3(e). One observes some "scattered" trapping 
effects, due to the interactions of the beads with the guides.

Next, the response of asymmetric systems was analyzed. For these tests the two branches of the Y-shaped guide were filled with particles of different materials, while the stems were always filled with stainless steel particles. The first of such systems was composed of branches filled with stainless steel and PTFE particles (Fig. 4). This particular arrangement was selected because the two materials have a well-known highly nonlinear response $[2,6,12,14,15]$ and a large difference in the signal speed. A single solitary wave was excited in the initial chain using a stainless steel bead as the striker. It is evident both from the experiments and the numerical results [Figs. 4(a) and 4(b) correspondingly] that once the excited pulse reaches the interface it splits asymmetrically in the two branches. The speed of the signal in the stainless steel branch is estimated to be $\sim 4$ times larger than the speed of pulse traveling in the softer PTFE branch. The system supports the propagation of a leading solitary waves in the two branches, although the properties of the solitary waves are different in the two branches. In addition the numerical results reports the presence of small amplitude reflections from the $120^{\circ}$ bends. The pulses formed in the softer PTFE section appear to remain trapped between the two stems and to "bounce" back and forth with a behavior similar to $[17,31]$. We confirmed this effect with force-density plots [Fig. 4(c)], showing the time history of numerically computed particle forces in the subsystem composed of stem 1 , PTFE branch and stem 2. This phenomenon can be useful for several practical applications ranging from acoustic delay lines and shock/energy trapping.

Results obtained from an interface composed of stainless steel and Parylene-C coated beads are reported in Figs. 5 and 6. Such system creates a special interface featuring particles with uniform mass but with different elastic moduli between the two branches. Experiments [Fig. 5(a)] showed that when a single incident solitary wave excited in the initial chain propagated through the first bifurcated branch, the signal split into two parts: one composed of a leading solitarylike pulse in the bare stainless steel branch [see second curve from the top in Fig. 5(a)]; and the other composed of a train of $\sim 4$ solitarylike pulses that propagated in the Parylene-C coated section [third curve from the top in Fig. 5(a)]. Such wave-train formation in the softer, but equally heavy, Parylene-C section was fairly unexpected. It could be caused by the complex dynamic response of the elastically softer polymeric coating, different from the assumed behavior of rigid particles with elastic modulus similar to Parylene-C (the numerical model used does not account for the dynamics of "layered" coating on the beads). The numerical analysis [Fig. 5(b)] developed for the coated particles assumed homogeneous beads with the mass of a steel particle and the elastic modulus of Parylene- $C$. The modeling is not able to capture entirely the particular multipulse effect observed in experiments, although showing an overall qualitative agreement with the experimental data. It is relevant to mention that both in the case of the impact with a small striker $(0.45$ $\mathrm{g}$ ), and in the case of the impact with a large striker $(5.33 \mathrm{~g})$, the peak values of the relative displacements calculated numerically between the beads of the Parylene-C branch were of the order of $10 \mu \mathrm{m}$ (with a coating thickness of $50 \mu \mathrm{m}$ ). The average values were of the order of $5 \mu \mathrm{m}$ in the case of the large striker, and of the order of $2 \mu \mathrm{m}$ in the case of the small striker. These values of displacements suggest that the contact interaction between particles remained confined to the coating layer.

Pulse trapping analog to the one reported for the PTFE case is also observed, as shown in Figs. 5(a) and 5(b) and in the density plot of Fig. 5(c). These results are relevant to underline the presence of self phase-shifting in the system and they suggest possible applications of such structures as delay lines, pulse scramblers and chaotization devices.

Analogous results were obtained when testing the same beads configuration exciting a train of solitary pulses in the first stem of the system [see Figs. 6(a)-6(c)]. In this case, the signal detected on the 5th particle in the stem 2 [bottom curve in Figs. 6(a) and 6(b)] shows clearly the separate arrival of the train of waves from the steel branch (at $\sim 0.6 \mathrm{~ms}$ from the triggering time) and from the Parylene coated section, at $\sim 1.4 \mathrm{~ms}$, triggering a "delay" effect on the pulse arrival. Besides the observation of the described incident and transmitted pulses, a complex dynamic "rattling" among the beads and reflections from the bends and the interfaces is also observed. This is evident in the signal detected by the sensors located in particle number 7 in the branches and in particle number 5 in the stem 2 [see Figs. 6(a) and 6(b)], and in the corresponding density plot [shown in Fig. 6(c)]. Such complex dynamic rattling and reflections from the holder are also the likely cause of the observed large amplitude secondary train of waves visible in the fourth curve of Fig. 6(a) corresponding to the gauge in 5 th particle in stem 2 . This unusually high amplitude signal was observed only in systems presenting nonsymmetric arrangement of particles in the two branches when such systems are excited with a largemass striker. We connect this behavior with possible particles misalignment in the experimental setup in the vicinity of the Y-junction interface. We plan to investigate this phenomenon in future publications.

\section{CONCLUSIONS}

The behavior of strongly nonlinear waves in twodimensional granular branches resembling typical forcechains present in three-dimensional assemblies was studied in this work. We describe the behavior of incident and transmitted pulses across interfaces and demonstrated experimentally the ability to bend, split, scramble, trap, and delay single and trains of solitary wavelike pulses. The presented results provide a simple model system for tailoring the behavior of pulses propagating in multidimensional systems composed from granular chains. In addition, we analyzed the behavior of a pulse propagating through the interface between steel and polymer coated steel beads and showed the unexpected decomposition of a single solitary wave into a train of $\sim 4-5$ separated pulses. The principles demonstrated here can be utilized for the design of new composite structures and devices. 


\section{ACKNOWLEDGMENTS}

C.D. acknowledges support from the Army Research Office (MURI grant U.S. ARO Grant No. W911NF-09-1-0436, Dr. David Stepp and Proposal No. 54272-EG, Dr. Bruce La-
Mattina). F.F. greatly acknowledges the support of the University Centre for Risk Prediction and Prevention (CUGRI), associating the Universities of Salerno and Napoli "Federico II," Italy. F.F. also thanks the Graduate Aerospace Laboratory at Caltech (GALCIT) for hospitality during his visit.
[1] V. F. Nesterenko, Prikl. Mekh. Tekh. Fiz. 24, 136 (1983); J. Appl. Mech. Tech. Phys. 24, 733 (1984).

[2] A. N. Lazaridi and V. F. Nesterenko, Prikl. Mekh. Tekh. Fiz. 26, 115 (1985); J. Appl. Mech. Tech. Phys. 26, 405 (1985).

[3] V. F. Nesterenko, Dynamics of Heterogeneous Materials (Springer-Verlag, New York, 2001), Chap. 1.

[4] V. F. Nesterenko, J. Phys. IV 4, C8 (1994).

[5] V. F. Nesterenko, A. N. Lazaridi, and E. B. Sibiryakov, Prikl. Mekh. Tekh. Fiz. 2, 19 (1995); J. Appl. Mech. Tech. Phys. 36, 166 (1995).

[6] C. Coste, E. Falcon, and S. Fauve, Phys. Rev. E 56, 6104 (1997).

[7] S. Sen, M. Manciu, and J. D. Wright, Phys. Rev. E 57, 2386 (1998).

[8] C. Coste and B. Gilles, Eur. Phys. J. B 7, 155 (1999).

[9] C. Daraio, V. F. Nesterenko, and S. Jin, APS-SCCM-2003, 197-200, AIP Conference Proceedings, Portland (Oregon), edited by M. D. Furnish, Y. M. Gupta, and J. W. Forbes (AIP, New York, 2004).

[10] J. Hong, Phys. Rev. Lett. 94, 108001 (2005).

[11] V. F. Nesterenko, C. Daraio, E. B. Herbold, and S. Jin, Phys. Rev. Lett. 95, 158702 (2005).

[12] S. Job, F. Melo, A. Sokolow, and S. Sen, Phys. Rev. Lett. 94, 178002 (2005).

[13] L. Vergara, Phys. Rev. Lett. 95, 108002 (2005).

[14] C. Daraio, V. F. Nesterenko, E. B. Herbold, and S. Jin, Phys. Rev. E 72, 016603 (2005).

[15] C. Daraio, V. F. Nesterenko, E. B. Herbold, and S. Jin, Phys. Rev. E 73, 026610 (2006).

[16] C. Daraio and V. F. Nesterenko, Phys. Rev. E 73, 026612 (2006).

[17] C. Daraio, V. F. Nesterenko, E. B. Herbold, and S. Jin, Phys. Rev. Lett. 96, 058002 (2006).
[18] M. A. Porter, C. Daraio, E. B. Herbold, I. Szelengowicz, P. G. Kevrekidis, Phys. Rev. E 77, 015601(R) (2008).

[19] E. B. Herbold, J. Kim, V. F. Nesterenko, S. Wang, and C. Daraio, Acta Mech. 205, 85 (2009).

[20] S. Y. Wang, E. B. Herbold, and V. F. Nesterenko, AIP Conference Proceedings No. 1227, Melville, New York, edited by J. D. Goddard, J. T. Jenkins, and P. Giovanale (AIP, New York, 2010).

[21] N. Boechler, G. Theocharis, S. Job, M. A. Porter, P. G. Kevrekidis, C. Daralo, Phys. Rev. Lett. 104, 244302 (2010).

[22] C. Goldenberg and I. Goldhirsch, Nature (London) 435, 188 (2005).

[23] E. I. Corwin, H. M. Jaeger, and S. R. Nagel, Nature (London) 435, 1075 (2005).

[24] T. S. Majmudar and R. P. Behringer, Nature (London) 435 , 1079 (2005).

[25] K. M. Roessig, J. C. Foster, Jr., and S. G. Bardenhagen, Exp. Mech. 42, 329 (2002).

[26] A. Shukla, C. Y. Zhu, and M. Sadd, J. Strain Anal. 23, 121 (1988).

[27] C. Daraio and V. F. Nesterenko, APS-Shock Compression of Condensed Matter, AIP Conference Proceedings, Big Island, Hawaii, CP955, edited by M. Elert, M. D. Furnish, R. Chau, N. Holmes, and J. Nguyen (AIP, New York, 2007), pp. 14191422.

[28] S. Job, F. Melo, A. Sokolow, and S. Sen, Granular Matter 10, 13 (2007)

[29] R. Carretero-González, D. Khatri, M. A. Porter, P. G. Kevrekidis, C. Daraio, Phys. Rev. Lett. 102, 024102 (2009).

[30] S. Goldstein, Classical Mechanics, 2nd ed. (Addison-Wesley, Reading, MA, 1980), Chap. 2.

[31] F. Fraternali, M. A. Porter, and C. Daraio, Mech. Adv. Mater. Struct. 17, 1 (2010). 